\title{
Density and Concentration Measurement Applications for Novel MEMS-based Micro Densitometer for Gas
}

\section{Anwendungen zur Dichte- und Konzentrationsmessung mit neuen MEMS-basierten Gasdichtesensoren}

\author{
Christof Huber ${ }^{1}$ \\ ${ }^{1}$ Endress+Hauser Flowtec AG, Kägenstrasse 7, CH-4153 Reinach, Switzerland
}

\begin{abstract}
This paper explores applications of recently released MEMS (Micro Electro Mechanical System) based process densitometer for gas. The core of the sensor is a resonating silicon microtube which is flowed through by the process gas. Due to the very low density of silicon and the fact that the tube is resonating in a vacuum cavity very good density sensitivity is achieved even for low fluid densities. The sensor therefore perfectly suits gas density applications with a medium pressure between 5 and 20 bar. The microfluidic sensor has density and temperature measurement capabilities. Additionally pressure is monitored along the fluidic path. From these measured physical properties, real time quality information of the measured gas such as molar mass, reference density, specific gravity, gas composition and calorific value can be derived. Process applications are demonstrated with experimental and theoretical results.
\end{abstract}

Keywords: gas quality, density, MEMS, concentration, natural gas

\section{Introduction}

In recent years Endress + Hauser $(\mathrm{E}+\mathrm{H})$ worked on the implementation and adaptation of the resonating tube measuring principle in a MEMS sensor by etching a micro tube structure from a silicon wafer $[1,2]$. Such micro machined silicon based resonating tube sensors allow now to consider applications for this measuring principle $[3,4]$.

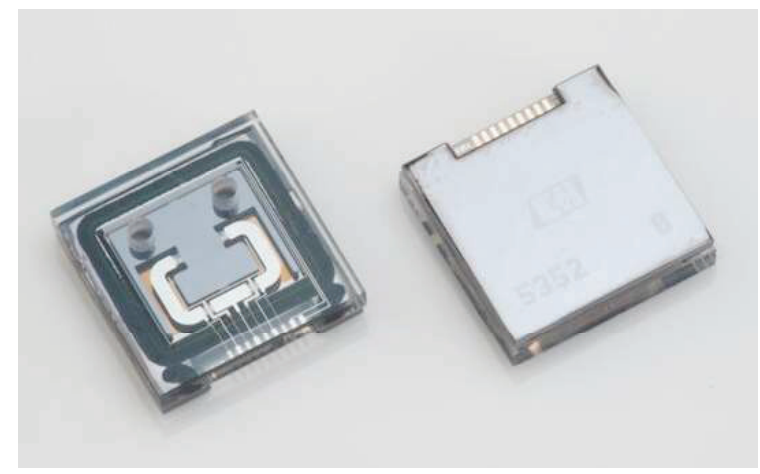

Fig. 1: $\quad$ E+H MEMS sensor chip. Left: bottom view with fluidic connection holes, sensing and excitation pads. Right: top view on the sensor cap and the bond pads for electrical connections.
2016 a process densitometer for gas using the $\mathrm{E}+\mathrm{H}$ MEMS-sensor chip was released [5]. The sensor has density, temperature and pressure measurement capabilities. An absolute density accuracy of $0.1 \mathrm{~kg} / \mathrm{m}^{3}$ can be achieved over a pressure range of 1 to 20 bar and from -20 to $60^{\circ} \mathrm{C}$. Thus, this makes it the perfect sensor for gas density measurement in medium pressure applications from about 5 to 20 bar. In this pressure range one can achieve a relative measuring accuracy of about $3 \%$ to $0.3 \%$.

From these measured physical properties, real time quality information of the measured gas, such as molar mass, reference density, specific gravity, gas composition and calorific value can be derived.

\section{Measuring Principle}

The frequency of a resonating tube depends on the stiffness $E \%$, and the total mass of the tube and the containing fluid. Density measurement methodology is rather simple in such a device, the higher the weight of the fluid in the filled tube, the lower the frequency $f$ according to eq. (1) 


$$
f=\text { const } \sqrt{\frac{E \cdot I}{m_{\text {tube }}+m_{\text {fluid }}}}
$$

Conventional process densitometers made from steel are typically not sensitive enough to accurately measure gas density at low pressures. A main advantage of a silicon tube over a conventional steel tube is its by 3.4 times lower material density. This leads to a significantly higher sensitivity of a silicon tube sensor for changes in the fluid density compared to a steel tube sensor with the same dimensions.

Another important factor for good density sensitivity is to reduce ambient influences on the oscillating tube, like an additional mass effect of the atmospheric air surrounding the tube. In our case, the microtube oscillates in a vacuum cavity in order to reduce squeeze-film damping [6]. This vacuum eliminates any additional mass effect on the tube and helps to increase the signal to noise ratio and density sensitivity for low pressure gases significantly.

\section{Concentration determination applications using gas density}

The value of micro gas sensors measuring accurately density in various applications will be shown in the course of this article.

When measuring in process on-line the temperature and the pressure have to be measured together with the density information. The density of a gas depends on its molar mass $M$, the actual pressure $p$ and temperature $T$ to eq. (2). $R$ is the universal gas constant. $Z$ is the real gas compressibility factor of the specific gas or gas mixture. $Z$ depends on temperature and pressure. For an ideal gas $Z$ equals 1 .

$\rho=\frac{M \cdot p}{Z \cdot R \cdot T}$

A typical application is to determine the concentration of individual components in a gas mixture.

The density of a mixture of gases is defined by eq. (3) where $x_{i}$ is the molar fraction of the component i.

$\rho=\frac{\sum_{i=1}^{N} x_{i} M_{i} \cdot p}{Z_{m i x} \cdot R \cdot T}$

From eq. (3) can be seen that it is not possible to get the exact concentration information or a mixture of more than two gases, if only density, temperature and pressure are known.
However for a binary mixture of two known gases it works as shown in eq. (4) and (5).

$$
\begin{aligned}
& \rho=\frac{\left[x_{1} M_{1}+\left(1-x_{1}\right) M_{2}\right] \cdot p}{Z_{\text {mix }} \cdot R \cdot T} \\
& x_{1}=\frac{\rho \cdot Z_{\text {mix }} \cdot R \cdot T-M_{2} p}{\left[M_{1}-M_{2}\right] p}
\end{aligned}
$$

Eq. (4) and eq. (5) illustrate that the larger the difference in molar mass of the two components the more sensitive the density information for concentration changes becomes.

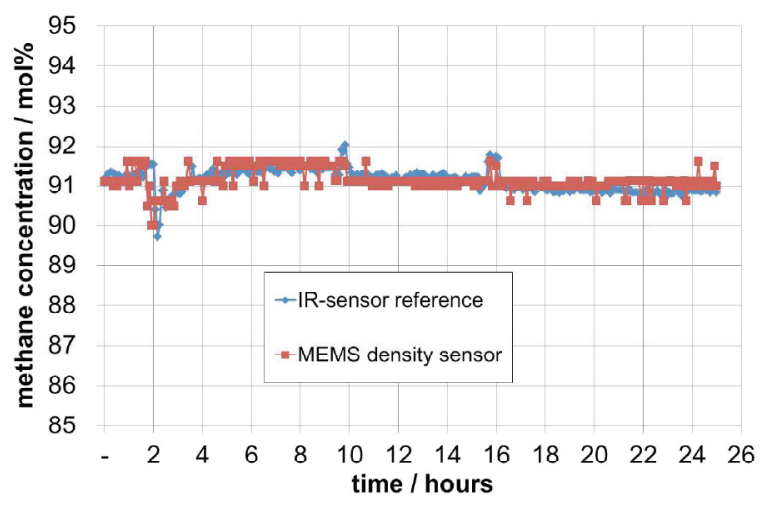

Fig. 2: Continuous biogas measurement over 1 day with two different sensing technologies (IR-adsorbtion versus density measurement). The accuracy of the concentration determination of methane and carbon dioxide using the MEMS density sensor is expected to be better $\pm 0.5 \%$. Measuring pressure was about 15 bar. The data of the two sensors correspond nicely within the specifications.

A nice example of a binary gas mixture is biogas. After processing it consists mainly of methane and carbon dioxide. Fig. 2 shows a continuous biogas measurement over a time period of 25 hours. An IR-adsorption sensor was used as a reference. The accuracy of the concentration determination of methane and carbon dioxide using the MEMS density sensor is expected to be better $\pm 0.5 \%$ while measuring at a pressure of about 15 bar. The data of the two sensors correspond nicely with these specifications. 


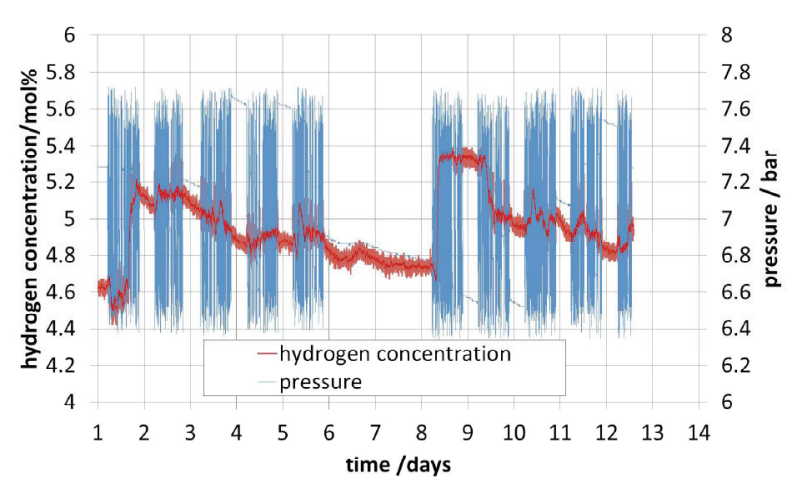

Fig. 3: Continuous monitoring of welding gas (hydrargon) in a production facility. The accuracy of the concentration determination of hydrogen in argon using the MEMS density sensor is expected to be better than $\pm 0.1 \%$. The measuring pressure varied between 6.4 and 7.7bar The hydrogen concentration in this case should be constant at $5 \%$. However, as it can be seen from the measurements there is some periodic variation in concentration.

Another example is the monitoring of welding gas (hydrargon) in a production facility. Usually the hydrogen concentration is not monitored. The hydrargon mixture is produced in place by blending pure argon und hydrogen using highly stable pressure regulators and mixing valves. In this application the hydrogen content should be constant at $5 \%$. However, as it can be seen easily from the measurements that there is some periodic variation in concentration ranging from $4.6 \%$ to $5.4 \%$ over a week. The accuracy of the concentration determination of hydrogen in argon using the MEMS density sensor is expected to be better than $\pm 0.1 \%$. Measuring pressure varied between 6.4 and $7.7 \mathrm{bar}$ during the measurement. This has no influence on the concentration output, since pressure as well as temperature are measured and compensated in the concentration calculations according to eq. (5).

These were only two examples for gas quality monitoring were density measurement can bring a benefit. There are of course a lot of different similar binary mixture applications.

\section{Natural gas applications using gas density}

Another field of application is measuring and monitoring gas mixtures for combustion. The most popular of these mixtures is natural gas. Natural gas is not a binary mixture. It consists of hydrocarbon gases like methane, ethane, propane, butane, pentane, etc. and inert gases like nitrogen, carbon dioxide and helium. The exact composition varies from one source to another. In fig. 4 typical composition ranges of natural gas are shown.

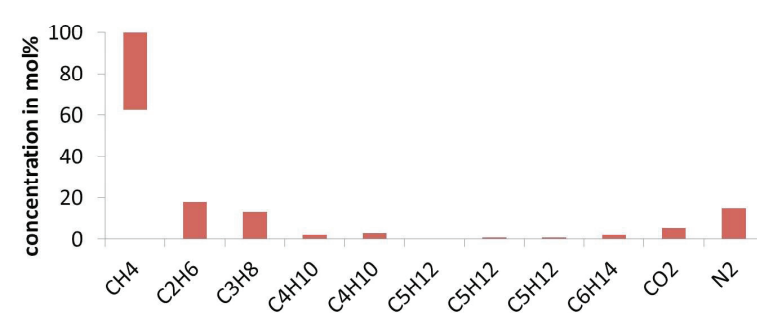

Fig. 4: Typical composition ranges of natural gas.

Renewable energies become more and more popular. Therefore also significant amounts of biogas (methane from fermentation of organic material) or hydrogen (produced by the electrolysis of water using electric power from wind turbines) can be found in natural gas networks.

An accurate determination of the molar composition of every single gas component is only possible with chromatographic methods (GC). However GCs are bulky, expensive, need maintenance, calibration and reference gases. Furthermore, the measurement is not continuous. A measuring cycle takes several minutes.

What can a density measurement offer instead for natural gas applications? The resonating MEMS sensor is measuring continuously. The sampling rate is $>1 \mathrm{~Hz}$. Hence measuring data in real time can be used to control a process, an engine or a burner. The direct measuring value is the actual density, of the natural gas sample in the measuring cell at actual measuring conditions i.e. temperature and pressure. Sometimes actual density is really needed for example for the conversion of the output of a volume flow measuring device in mass flow. However, the actual density is often not a significant measure for the gas quality because it depends strongly on temperature and pressure. Other parameters are usually of higher value like the average molar mass, reference density or the specific gravity at reference conditions. This information can be deduced from the measured triple density, temperature and pressure using specific correlation methods for natural gas. The feasibility of such a correlation method for typical natural gas mixtures (fig. 4) using density measurement in a temperature range of 0 to $60^{\circ} \mathrm{C}$ and a pressure range from 1 to 20 bar is shown in fig, 5 . The accuracy for a 5 to $95 \%$ confidence interval is $\pm 0.3 \%$. Reference density or specific gravity can be derived from av. molar mass. 


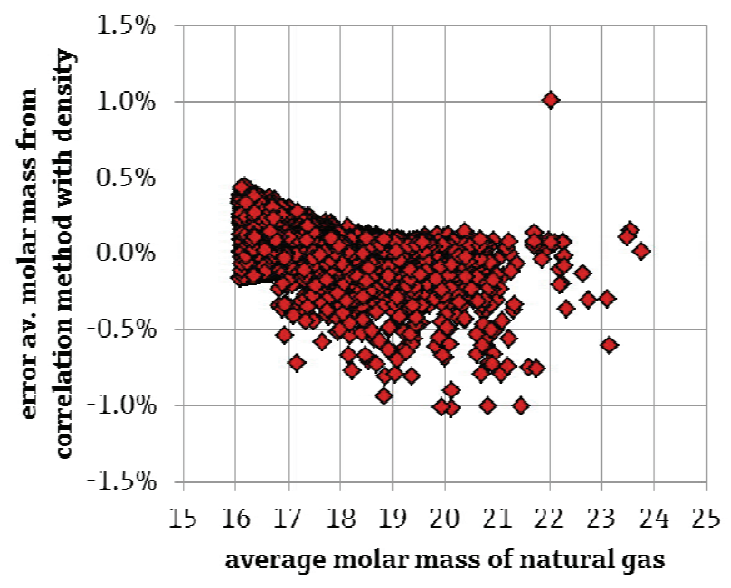

Fig. 5: Accuracy of a correlation method for average molar mass of typical natural gas mixtures (fig 5.) using density measurement in a temperature range of 0 to $60^{\circ} \mathrm{C}$ and a pressure range from 1 to 20 bar. (5 to 95\% confidence interval $\pm 0.3 \%$ )

Taking density accuracy of the sensor into account it can be stated, that MEMS density measurement is able to deduce real time av. molar mass or reference density of natural gas with an accuracy of about $\pm 1-2 \%$. Av. molar mass can be used to correlate other physical properties of the gas mixture such as calorific value or Wobbe index

The calorific value is probably the most important quality parameter of combustible gas. In case pure of fuel gas mixtures including hydrocarbon gases and hydrogen av. molar mass can be correlated directly with its calorific value, regardless if gross calorific value or net calorific value is considered. However, inert gases will add a significant error to that model correlation if present in the examined gas mixture as can be seen from fig. 6 .

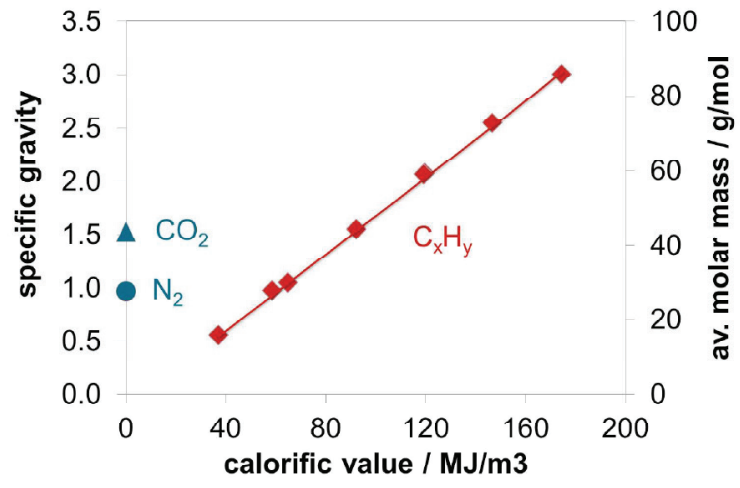

Fig. 6: Calorific value of pure hydrocarbon gases (red) versus molar mass or specific gravity, respectively. The perfect linear correlation of energy content and density is obvious. However, inert gases like nitrogen and carbon dioxide (blue) do not follow this relationship.

Hence if applying the hydrocarbon gas correlation from fig. 6 to a real natural gas mixture it will become more and more inaccurate the higher the total inert gas content becomes. The relationship between calorific value error and total inert gas content is shown in fig. 7. Errors can be very large; however the connection is strongly linear. If the total inert gas concentration is known the expected error of calorific value is also known and can be corrected according to eq. (6). It should be reminded that this finding is of general nature also valid other gas density measuring principles.

error CV / \% = 1.6 $\cdot$ total inert gas $/ \%$

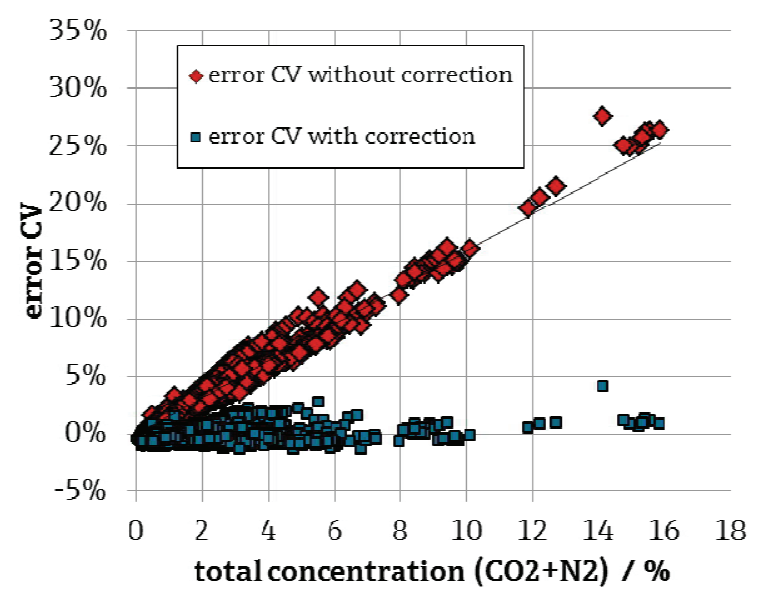

Fig. 7: Red diamonds: error of calorific value of natural gas deduced from average molar mass using the linear relationship for hydrocarbon gases from fig 6. Blue squares: same data with linear inert gas correction.

All the above calculations were done for natural gas mixtures indicated in fig. 4. and using the NIST reference fluid thermodynamic and transport properties database [7]. Similar correlations can be found for other gases such as blended natural gas compositions containing significant amounts of hydrogen or fuel gas where hydrogen and methane are the main components.

\section{Conclusions and outlook}

Resonating MEMS-based gas density sensors can be used to measure on-line density at process conditions. Density sensors can be calibrated whit traceable fluids in the factory. Hence, they are measuring absolute density 
and the use reference gases in the field is not necessarily needed.

By measuring the density, temperature and pressure of a gas one can deduce exact composition of binary gas mixtures, such as methane/carbon dioxide, argon/hydrogen or methane/propane. Examples have been shown where the absolute concentration accuracy is better than $1 \mathrm{~mol} \%$ even better than $0.1 \mathrm{~mol} \%$ depending mainly on the molar mass difference of the two involved components and the available process pressure.

When measuring a more complex gas mixture like natural gas or fuel gas, correlation methods have to be used in order to derive information of the gas mixture like the average molar mass, the reference density or the specific gravity at reference conditions. The accuracy of these derived physical properties can be better than $2 \%$, depending on the process conditions.

The determination of caloric value of the natural or fuel gas is possible, but its measuring accuracy depends strongly on the present inert gas content.

A potential solution to this problem would be to measure additional physical properties of the gas mixture. $\mathrm{E}+\mathrm{H}$ was investigating the capabilities of a resonating silicon microcantilever knows for its use in atomic force microscopy (AFM) as sensing element for low gas densities at atmospheric pressure [8]. The new sensor concept is able to measure gas density and viscosity simultaneously with a relative accuracy of $1 \%$ even at atmospheric pressure. Similar studies were done by other groups showing the high potential of resonating micro- cantilever [9] or micro-tuning forks [10] measuring gas density and viscosity. The additional viscosity information could potentially help to overcome the inert gas problem. Such new sensor concepts could add benefit to the analysis of more component mixtures. This will be investigated further.

\section{Acknowledgments}

The author likes to acknowledge the work and support from J. Knall from Endress+Hauser Flowtec AG and the colleagues from TrueDyne Sensors AG specially P. Reith, H. Feth and A. Rasch.

\section{Bibliography}

[1] Zhang Y, Tadigadapa S, Najafi N. A micromachined Coriolis-force-based mass flowmeter for direct mass flow and fluid density measurement. Transducer '01 Eurosensors XV, the 11th international conference on solid-state sensors and actuators. Munich, Germany (2001)
[2] Sparks D, Smith R, Cripe J, Schneider R, Najafi N. A portable MEMS Coriolis mass flow sensor. Proc. IEEE Sensors 2003 (IEEE Cat. No.03CH37498) 1(8),337-339 (2003) doi:10.1109/ICSENS.2003.1278953.

[3] Huber C., Touzin, M, New MEMS-based microcoriolis density measurement technology, Proceedings of the ASME 2011 9th International Conference on Nanochannels, Microchannels, and Minichannels, Edmonton, Alberta, CANADA, (2011) ICNMM2011-58030

[4] Huber C, M, MEMS-based Micro-Coriolis Density and Flow Measurement Technology, AMA Conference, (2015) doi: 10.5162/sensor2015/B6.1

[5] http://www.endress.com/

[6] Sparks D., Smith R., Schneider R., Cripe J., Massoud-Ansari S., Chimbayo A., Najafi N., A variable temperature, resonant density sensor made using an improved chip-level vacuum package, Sensors and Actuators A, 107, 119124 (2003)

[7] NIST National Institute of Standards and Technology, Reference Fluid Thermodynamic and Transport Properties Database www.nist.gov/srd/nist23.cfm

[8] Badarlis A, Pfau A., Kalfas A., Gas Density and Viscosity Measurement Using Micro-Cantilerver Sensor, AMA Conference, (2015) doi: 10.5162/sensor2015/B6.3

[9] Boskovic S., Chon J.W.M., Mulvaney P., Sader J.E, Rheological measurements using microcantilevers, Journal of Rheology, 46(4), 891 (2002)

[10] Sell J.K., Niedermayer A.O., Jakoby B., Simultaneous measurement of density and viscosity in gases with a quartz tuning fork resonator by tracking of the series resonance frequency, Procedia Engineering, 25, 12971300, (2011) 